\title{
IGG4 DISEASE WITH GLOMERULAR INVOLVEMENT MIMICKING ANCA-ASSOCIATED VASCULITIS: A CASE REPORT
}

\author{
Carolina Dias da Silva Amorim ${ }^{1}$, Carlos Eduardo Lopes Tavares de Meloํㅡ, Glauber Ruan Nelson Bem ${ }^{1}$, Jonas Braynner ${ }^{1}$, Rafaela \\ Silva Guimarães Gonçalves ${ }^{1, \star}$, Henrique de Ataíde Mariz ${ }^{1}$
}

1.Universidade Federal de Pernambuco, Recife (PE), Brazil.

*Corresponding author: rafa_sgg@hotmail.com

\section{BACKGROUND}

IgG4-related disease (IgG4-RD) is considered to involve conditions of systemic inflammatory fibrosis, including autoimmune pancreatitis, retroperitoneal fibrosis, chronic sclerosing cholangitis and inflammatory pseudotumor. IgG4-RD has now been reported in nearly every organ, though it was first identified in the pancreas and salivary glands. This disease manifests as organ enlargement or nodular lesions in various organs concurrently or asynchronous as a result of marked infiltration of lymphocytes and lgG4-positive plasma cells as well as fibrosis. IgG4-related kidney disease (IgG4-RKD) mainly manifests as plasma cell-rich tubulointerstitial nephritis with increased lgG4+ plasma cells and fibrosis. However, we report a rare case of IgG4-RKD involving the glomerulus.

\section{CASE REPORT}

A 42-year-old man presenting with macroscopic hematuria for 5 days and fatigue. At admission, he had nephritic syndrome with his urine analyses showing dysmorphic hematuria and $2.575 \mathrm{mg}$ of proteinuria. His medical history showed asthma and two years follow-up with otolaryngologist because of rhinorrhea, nasal obstruction and anosmia which he was treated with antiallergic drugs and corticosteroids with little improvement. One year ago, he had eyelid edema that evolved to exophthalmos. At admission he also reported weight loss (12 kg in 10 months) and a tumor in cervical region. His physical examination revealed exophthalmos and palpable lymph nodes at cervical region (Figure 1). Chest computed tomography (Figure 2) showed bilateral bronchial thickening. Face image showed filling with soft tissues density of the frontal, ethmoidal and maxillary sinuses and presence of erosion on frontal sinus (Figure 1). Nuclear magnetic resonance (Figure 3) showed lacrimal gland enlargement with infiltration of ocular extrinsic muscle, promoting proptosis. A blood analysis revealed anemia (11.1), normal thyroid function, normal lymphocytes with 2,494 eosinophils, negative serology for syphilis, HIV and hepatitis, but c-ANCA was positive (anti-PR3). Nasal biopsy was inconclusive showing reaction lymphocytes follicles suggesting lymphoepithelial sialadenitis. Another biopsy was done in submandibular gland which then confirmed lgG4 disease (Figure 4). Corticosteroids was prescribed as methylprednisolone $500 \mathrm{mg}$ for 3 days and after $0.5 \mathrm{mg} / \mathrm{kg}$ with improvement of exophthalmos (Figure 5) and reduction in the size of the lacrimal and submandibular glands and improvement in proteinuria (160 mg/24 h).

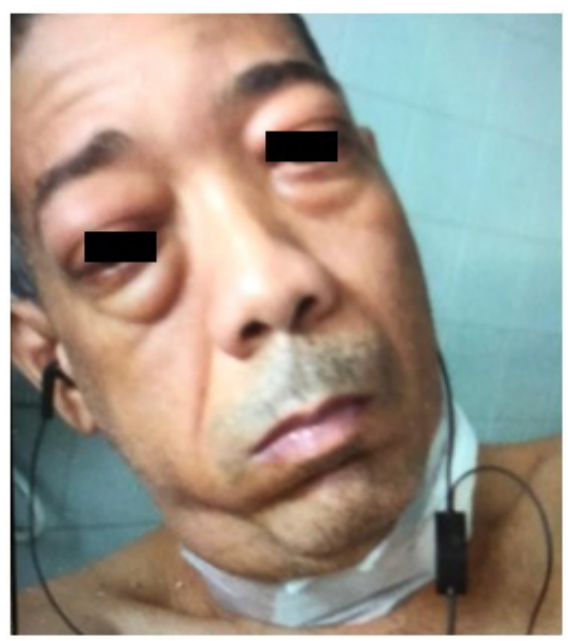

Figure 1. Exophthalmos and enlarged lymph node at cervical region.

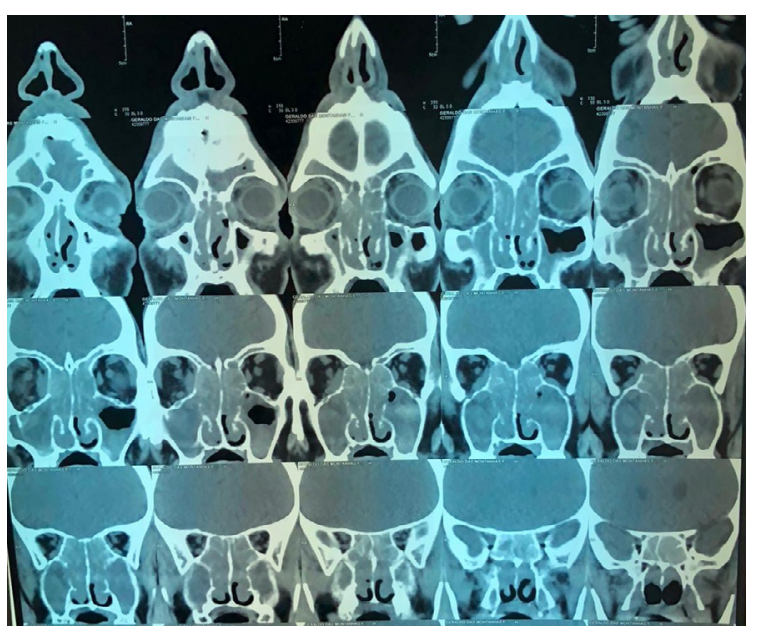

Figure 2. Computed tomography showing heterogeneous and hyperdense material, occupying the frontal, maxillary and sphenoid sinuses, as well as ethmoid cells, nasal cavities and upper portions of the nasopharynx. Several areas of blurring/lysis of the different bone boundaries coexist.

Realização: 

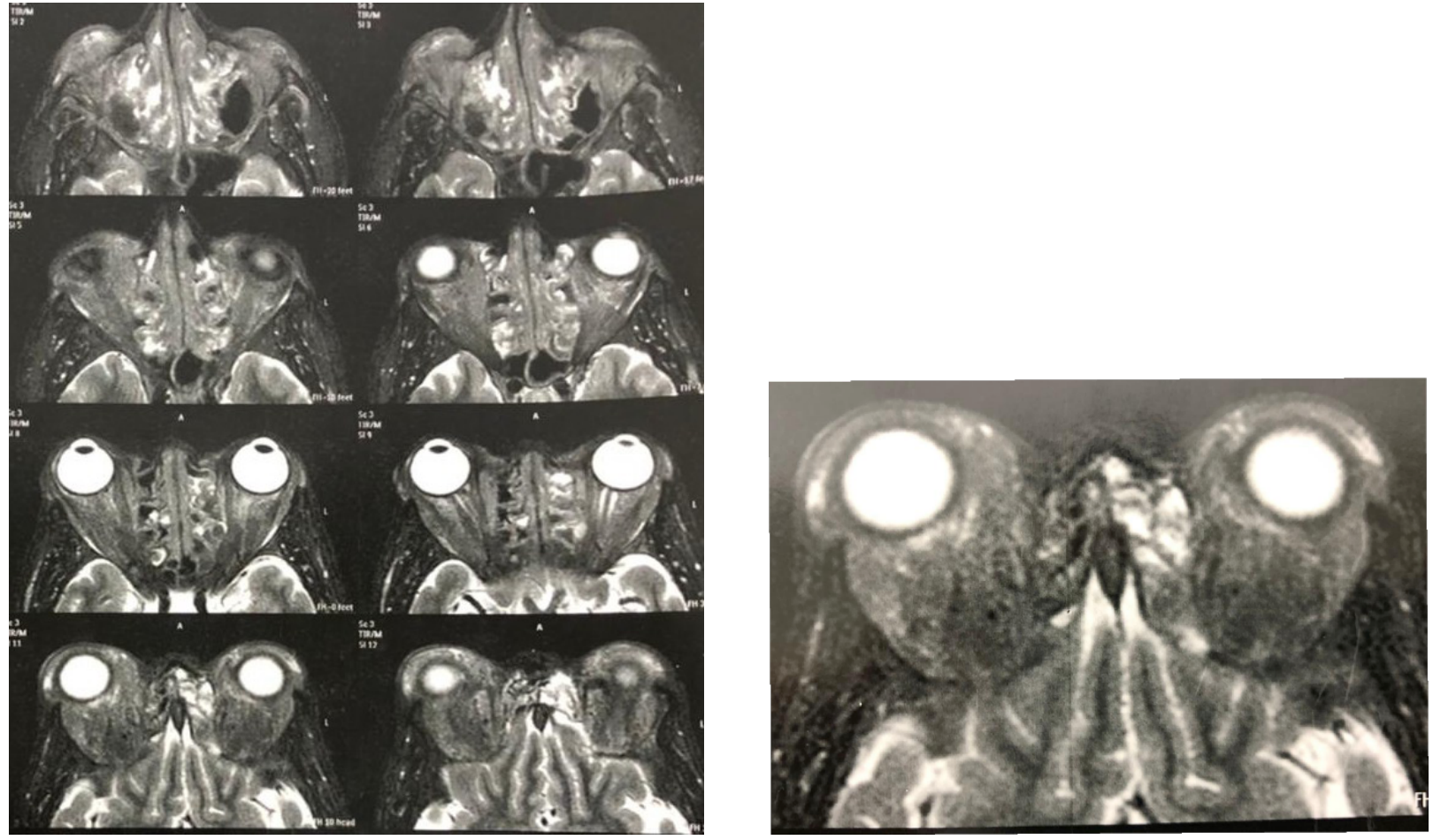

Figure 3. MRI: There is an important enlargement of the lacrimal glands, isointense on T1 and low signal on T2, bilateral with marked hypoattenuation of extrinsic ocular muscles, enlargement of the insertion of the superior rectum, with infiltration of these by anomalous tissue, more evident on the right promoting proptosis that suggests infiltrative disorder

SUBMANDIBULAR GLAND BIOPSY: PRESENCE OF ABOUT 80-90 IGG4 POSITIVE CELLS PER HIGH POWER FIELD, WITH AN IgG4/IgG RATIO OF 90

\begin{tabular}{|c|c|c|c|}
\hline Antibodies & Clone & Result & Block \\
\hline Human IgG & A57H & Positive & 60256 \\
\hline Imunoglobulin IgG4 & HP6025 & Positive & 60256 \\
\hline
\end{tabular}
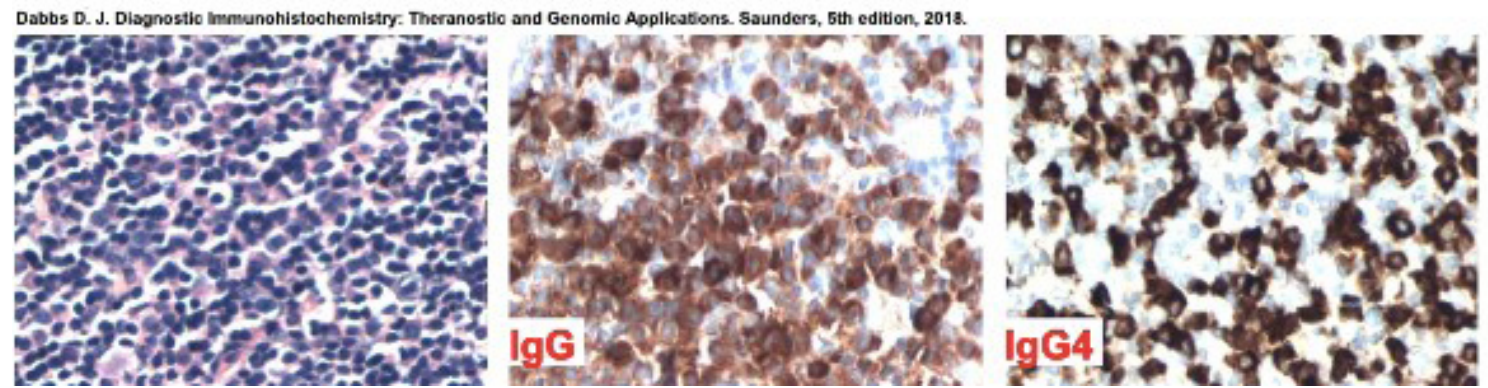

Figure 4. Salivary gland biopsy. 


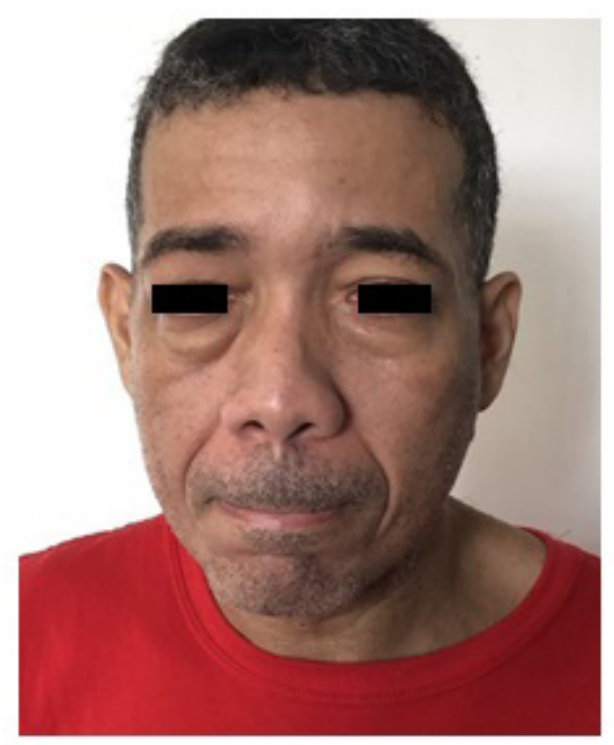

Figure 5. Improvement of exophthalmos and lymph node after corticosteroid treatment.

\section{CONCLUSION}

We report a case of IgG4-RD with glomerular involvement and eosinophilia mimicking ANCA-associated vasculitis, with fast and good steroid response, confirmed just after second biopsy.

\section{KEYWORDS}

IgG4-related disease, ANCA-associated vasculitis, C-ANCA. 